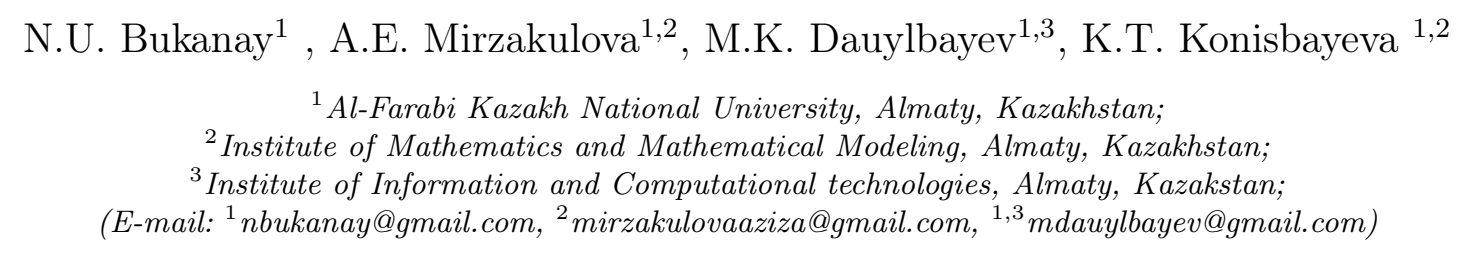

\title{
A boundary jumps phenomenon in the integral boundary value problem for singularly perturbed differential equations
}

\begin{abstract}
The article is devoted to the study of the asymptotic behavior of solving an integral boundary value problem for a third-order linear differential equation with a small parameter for two higher derivatives, provided that the roots of the "additional characteristic equation" have opposite signs. In the work are constructed the fundamental system of solutions, boundary functions for singularly perturbed homogeneous differential equation and are provided their asymptotic representations. An analytical formula of solution for a given singularly perturbed integral boundary value problem is obtained. Theorem about asymptotic estimates of solution is proved. For a singularly perturbed integral boundary value problem, the growth of the solution and its derivatives at the boundary points of this segment is obtained when the small parameter tends to zero. It is established that the solution of a singularly perturbed integral boundary value problem has initial jumps at both ends of this segment. In this case, we say that there is a phenomenon of boundary jumps, which is a feature of the considered singularly perturbed integral boundary value problem. Moreover, the orders of initial jumps were different. Namely, at the point $t=0$, there is a phenomenon of the initial jump of the first order, and at the point $t=1$, the order of the initial jump was equal to zero. The results obtained allow us to construct uniform asymptotic expansions of solutions of nonlinear singularly perturbed integral boundary value problems.
\end{abstract}

Keywords: singularly perturbed differential equation, asymptotic estimates, boundary functions, small parameter.

\section{Introduction}

Equations containing a small parameter in the highest derivatives are called singularly perturbed equations. Such equations are mathematical models of many applied problems. A significant contribution to the development of the theory of singularly perturbed equations was made by L. Schlesinger [1], G.D. Birkhoff [2], P. Noaillon [3], W. Wasow [4], A.N. Tikhonov [5, 6], M.I. Vishik, L.A. Lyusternik [7, 8], N.N. Bogolyubov, U.A. Mitropolsky [9], A.B. Vasilieva and V.F. Butuzov [10], Trenogin, V.A. [11], R.E. O'Malley [12], W. Eckhaus [13], K.W. Chang and F.A. Howes [14], J. Kevorkian and J.D. Cole [15], P.V. Kokotovic [16], S.A. Lomov [17], M.I. Imanaliev [18], K.A. Kassymov [19-21] and others.

Initial problems with singular initial conditions for a second-order nonlinear ordinary differential equation with a small parameter were first studied by M.I. Vishik and L.A. Lyusternik [8] and K.A. Kassymov [20]. They showed that the solution of the original problem with initial values leads to the solution of a degenerate equation with altered initial conditions when a small parameter approaches zero. Such problems became known as Cauchy problems with initial jumps. The most general cases of the Cauchy problem for singularly perturbed nonlinear systems of ordinary differential and integrodifferential equations, as well as for differential equations in partial derivatives of a hyperbolic type, was studied by K.A. Kassymov. Then, singularly perturbed initial and boundary value problems with initial jumps have been studied in [22-30]. In this paper, we consider general integral boundary value problems for linear ordinary differential equations of the third order with a small parameter with two highest derivatives, when the roots of an additional characteristic equation have opposite signs. It is shown that there is a phenomenon of boundary jumps. Boundary value problems without integral boundary conditions for singularly perturbed differential and integro-differential equations have been considered in [31-33]. 


\section{Statement of the problem and preliminaries}

Consider the singularly perturbed differential equation

$$
L_{\varepsilon} y \equiv \varepsilon^{2} y^{\prime \prime \prime}+\varepsilon A_{0}(t) y^{\prime \prime}+A_{1}(t) y^{\prime}+A_{2}(t) y=F(t),
$$

with integral boundary conditions

$$
\begin{aligned}
& h_{1} y(t, \varepsilon) \equiv y(0, \varepsilon)-\int_{0}^{1} \sum_{i=0}^{1} a_{i}(x) y^{(i)}(x, \varepsilon) d x=\alpha_{0}, \\
& h_{2} y(t, \varepsilon) \equiv y^{\prime}(0, \varepsilon)-\int_{0}^{1} \sum_{i=0}^{1} b_{i}(x) y^{(i)}(x, \varepsilon) d x=\alpha_{1}, \\
& h_{3} y(t, \varepsilon) \equiv y(1, \varepsilon)-\int_{0}^{1} \sum_{i=0}^{1} c_{i}(x) y^{(i)}(x, \varepsilon) d x=\beta,
\end{aligned}
$$

where $\varepsilon>0$ is a small parameter, $\alpha_{0}, \alpha_{1}, \beta$ are known constants independent of $\varepsilon$.

We will need the following assumptions:

C1) $A_{i}(t) \in C^{2}[0,1], i=\overline{0,2}, F(t) \in C[0,1]$.

$\mathrm{C} 2)$ The roots $\mu_{i}(t), i=1,2$ of "additional characteristic equation" $\mu^{2}(t)+A_{0}(t) \mu(t)+A_{1}(t)=0$ satisfy the following inequalities $\mu_{1}(t) \leq-\gamma_{1}<0, \quad \mu_{2}(t) \geq \gamma_{2}>0$.

C3)

$$
\bar{\Delta} \equiv y_{20}(1) a_{1}(1)\left(y_{30}(1)-\int_{0}^{1} \sum_{i=0}^{1} c_{i}(x) y_{30}^{(i)}(x) d x\right)+y_{20}(1)\left(1-c_{1}(1)\right) \cdot\left(1-\int_{0}^{1} \sum_{i=0}^{1} a_{i}(x) y_{30}^{(i)}(x) d x\right) \neq 0 .
$$

We consider homogeneous singularly perturbed equation associated with (1)

$$
L_{\varepsilon} y \equiv \varepsilon^{2} y^{\prime \prime \prime}+\varepsilon A_{0}(t) y^{\prime \prime}+A_{1}(t) y^{\prime}+A_{2}(t) y=0 .
$$

The system of fundamental solutions of the homogeneous singular perturbed differential equation (3) is as follows

$$
\begin{gathered}
y_{1}^{(q)}(t, \varepsilon)=\frac{1}{\varepsilon^{q}} \exp \left(\frac{1}{\varepsilon} \int_{0}^{t} \mu_{1}(x) d x\right)\left(\mu_{1}^{q}(t) y_{10}(t)+O(\varepsilon)\right), \quad q=\overline{0,2}, \\
y_{2}^{(q)}(t, \varepsilon)=\frac{1}{\varepsilon^{q}} \exp \left(-\frac{1}{\varepsilon} \int_{t}^{1} \mu_{2}(x) d x\right)\left(\mu_{2}^{q}(t) y_{20}(t)+O(\varepsilon)\right), \quad q=\overline{0,2}, \\
y_{3}^{(q)}(t, \varepsilon)=y_{30}^{(q)}(t)+O(\varepsilon), \quad q=\overline{0,2},
\end{gathered}
$$

here $\mu_{1}(t), \mu_{2}(t)$ are roots of the additional characteristic equation $\mu^{2}(t)+A_{0}(t) \mu(t)+A_{1}(t)=0$, the functions $y_{i 0}(t), i=\overline{1,3}$ are defined by these problems

$$
y_{i 0}^{\prime}(t)+\frac{3 \mu_{i}(t) \mu_{i}^{\prime}(t)+A_{0}(t) \mu_{i}^{\prime}(t)+A_{2}(t)}{\mu_{i}^{2}(t)} y_{i 0}(t)=0, \quad y_{i 0}(0)=1, \quad i=1,2,
$$




$$
A_{1}(t) y_{30}^{\prime}(t)+A_{2}(t) y_{30}(t)=0, \quad y_{30}(0)=1
$$

The asymptotic formula of Wronskian consisting of a system of fundamental solutions is expressed as follows

$$
\begin{gathered}
W(t, \varepsilon)=\frac{1}{\varepsilon^{3}} \exp \left(\frac{1}{\varepsilon} \int_{0}^{t} \mu_{1}(x) d x-\frac{1}{\varepsilon} \int_{t}^{1} \mu_{2}(x) d x\right) . \\
\cdot\left(\mu_{1}(t) \mu_{2}(t)\left(\mu_{2}(t)-\mu_{1}(t)\right) y_{10}(t) y_{20}(t) y_{30}(t)+O(\varepsilon)\right) \neq 0 .
\end{gathered}
$$

Let's enter the following functions

$$
K_{0}(t, s, \varepsilon)=\frac{P_{0}(t, s, \varepsilon)}{W(s, \varepsilon)}, K_{1}(t, s, \varepsilon)=\frac{P_{1}(t, s, \varepsilon)}{W(s, \varepsilon)},
$$

where $P_{0}(t, s, \varepsilon), P_{1}(t, s, \varepsilon)$ are the third order determinant obtained from the Wronskian $W(s, \varepsilon)$ by replacing the third row with $y_{1}(t, \varepsilon), 0, y_{3}(t, \varepsilon)$ and $0, y_{2}(t, \varepsilon), 0$ respectively. Sum of $K_{0}(t, s, \varepsilon)$ and $K_{1}(t, s, \varepsilon)$ is the Cauchy function. Therefore, these functions have the following properties

1 . With respect to the variable $t$ satisfy equation (3), i.e.

$$
L_{\varepsilon} K_{0}(t, s, \varepsilon)=0, \quad L_{\varepsilon} K_{1}(t, s, \varepsilon)=0, \quad t \in[0,1], \quad t \neq s .
$$

2. When $t=s$ satisfy the conditions

$$
K_{0}(s, s, \varepsilon)+K_{1}(s, s, \varepsilon)=0, \quad K_{0}^{\prime}(s, s, \varepsilon)+K_{1}^{\prime}(s, s, \varepsilon)=0, \quad K_{0}^{\prime \prime}(s, s, \varepsilon)+K_{1}^{\prime \prime}(s, s, \varepsilon)=1 .
$$

By applying formulas (5), (6), for functions $K_{0}(t, s, \varepsilon), K_{1}(t, s, \varepsilon)$ are valid the following asymptotic representations as $\varepsilon \rightarrow 0$

$$
\begin{aligned}
K_{0}^{(i)}(t, s, \varepsilon) & =\varepsilon^{2}\left(\frac{y_{30}^{(i)}(t)}{A_{1}(s) y_{30}(s)}-\frac{\mu_{1}^{i}(t) y_{10}(t)}{\varepsilon^{i} \mu_{1}(s)\left(\mu_{2}(s)-\mu_{1}(s)\right) y_{10}(s)} \exp \left(\frac{1}{\varepsilon} \int_{s}^{t} \mu_{1}(x) d x\right)+O(\varepsilon)\right), \\
K_{1}^{(i)}(t, s, \varepsilon) & =\varepsilon^{2}\left(\frac{\mu_{2}^{i}(t) y_{20}(t)}{\varepsilon^{i} \mu_{2}(s)\left(\mu_{2}(s)-\mu_{1}(s)\right) y_{20}(s)} \exp \left(-\frac{1}{\varepsilon} \int_{t}^{s} \mu_{2}(x) d x\right)+O(\varepsilon)\right), t \leq s, i=\overline{0,2} .
\end{aligned}
$$

Let functions $\Phi_{i}(t, \varepsilon), i=\overline{1,3}$ are solutions of the following problem

$$
L_{\varepsilon} \Phi_{i}(t, \varepsilon)=0, \quad i=\overline{1,3}, \quad h_{k} \Phi_{i}(t, \varepsilon)=\delta_{k i}, \quad k=\overline{1,3},
$$

where $\delta_{k i}$ is Kronecker symbol.

Functions $\Phi_{i}(t, \varepsilon), i=\overline{1,3}$ are called boundary functions and can be represented in the form

$$
\Phi_{i}(t, \varepsilon)=\frac{\Delta_{i}(t, \varepsilon)}{\Delta(\varepsilon)}, \quad i=\overline{1,3}
$$

where

$$
\Delta(\varepsilon)=\left|\begin{array}{lll}
h_{1} y_{1}(t, \varepsilon) & h_{1} y_{2}(t, \varepsilon) & h_{1} y_{3}(t, \varepsilon) \\
h_{2} y_{1}(t, \varepsilon) & h_{2} y_{2}(t, \varepsilon) & h_{2} y_{3}(t, \varepsilon) \\
h_{3} y_{1}(t, \varepsilon) & h_{3} y_{2}(t, \varepsilon) & h_{3} y_{3}(t, \varepsilon)
\end{array}\right|,
$$

$\Delta_{i}(t, \varepsilon)$ is the determinant obtained from $\Delta(t, \varepsilon)$ by replacing the $i$-th row by the fundamental system of solutions $y_{1}(t, \varepsilon), y_{2}(t, \varepsilon), y_{3}(t, \varepsilon)$ of the equation $L_{\varepsilon} y=0$. By taking account formulas (2), (4), we get asymptotic representation for determinant $\Delta(\varepsilon)$ :

$$
\Delta(\varepsilon)=\frac{1}{\varepsilon}\left(\mu_{1}(0) \bar{\Delta}+O(\varepsilon)\right)
$$

where $\bar{\Delta}$ has the form as in condition (C3). 
For boundary functions $\Phi_{i}^{(j)}(t, \varepsilon), j=\overline{0,2}, i=\overline{1,3}$ from (9) in view (4), (10) we obtain asymptotic representation as $\varepsilon \rightarrow 0$ :

$$
\begin{aligned}
& \Phi_{1}^{(j)}(t, \varepsilon)=\frac{1}{\varepsilon^{j-1}} \exp \left(\frac{1}{\varepsilon} \int_{0}^{t} \mu_{1}(x) d x\right) \frac{\mu_{1}^{j}(t) y_{10}(t) M_{11}}{\mu_{1}(0) \bar{\Delta}}- \\
& -\frac{1}{\varepsilon^{j}} \exp \left(-\frac{1}{\varepsilon} \int_{t}^{1} \mu_{2}(x) d x\right) \frac{\mu_{2}^{j}(t) y_{20}(t)\left(y_{30}(1)-\int_{0}^{1} \sum_{i=0}^{1} c_{i}(x) y_{30}^{(i)}(x) d x\right)}{\bar{\Delta}}+ \\
& +\frac{y_{20}(1)\left(1-c_{1}(1)\right) y_{30}^{(j)}(t)}{\bar{\Delta}}+O\left(\varepsilon+\frac{1}{\varepsilon^{j-2}} \exp \left(\frac{1}{\varepsilon} \int_{0}^{t} \mu_{1}(x) d x\right)+\frac{1}{\varepsilon^{j-1}} \exp \left(-\frac{1}{\varepsilon} \int_{t}^{1} \mu_{2}(x) d x\right)\right), \\
& \Phi_{2}^{(j)}(t, \varepsilon)=\frac{1}{\varepsilon^{j-1}} \exp \left(\frac{1}{\varepsilon} \int_{0}^{t} \mu_{1}(x) d x\right) \frac{\mu_{1}^{j}(t) y_{10}(t)}{\mu_{1}(0)}+\frac{1}{\varepsilon^{j-1}} \exp \left(-\frac{1}{\varepsilon} \int_{t}^{1} \mu_{2}(x) d x\right) \frac{\mu_{2}^{j}(t) y_{20}(t) M_{22}}{\mu_{1}(0) \bar{\Delta}}- \\
& -\varepsilon \frac{M_{23} y_{30}^{(j)}(t)}{\mu_{1}(0) \bar{\Delta}}+O\left(\varepsilon^{2}+\frac{1}{\varepsilon^{j-2}} \exp \left(\frac{1}{\varepsilon} \int_{0}^{t} \mu_{1}(x) d x\right)+\frac{1}{\varepsilon^{j-2}} \exp \left(-\frac{1}{\varepsilon} \int_{t}^{1} \mu_{2}(x) d x\right)\right), \quad j=\overline{0,2}, \\
& \Phi_{3}^{(j)}(t, \varepsilon)=\frac{1}{\varepsilon^{j-1}} \exp \left(\frac{1}{\varepsilon} \int_{0}^{t} \mu_{1}(x) d x\right) \frac{\mu_{1}^{j}(t) y_{10}(t) M_{31}}{\mu_{1}(0) \bar{\Delta}}+ \\
& +\frac{1}{\varepsilon^{j}} \exp \left(-\frac{1}{\varepsilon} \int_{t}^{1} \mu_{2}(x) d x\right) \frac{\mu_{2}^{j}(t) y_{20}(t)\left(1-\int_{0}^{1} \sum_{i=0}^{1} a_{i}(x) y_{30}(x)^{(i)} d x\right)}{\bar{\Delta}}+ \\
& +\frac{a_{1}(1) y_{20}(1) y_{30}^{(j)}(t)}{\bar{\Delta}}+O\left(\varepsilon+\frac{1}{\varepsilon^{j-2}} \exp \left(\frac{1}{\varepsilon} \int_{0}^{t} \mu_{1}(x) d x\right)+\frac{1}{\varepsilon^{j-1}} \exp \left(-\frac{1}{\varepsilon} \int_{t}^{1} \mu_{2}(x) d x\right)\right),
\end{aligned}
$$

where

$$
\begin{aligned}
& M_{11}=\left|\begin{array}{cc}
-b_{1}(1) y_{20}(1) & y_{30}^{\prime}(0)-\int_{0}^{1} \sum_{i=0}^{1} b_{i}(x) y_{30}^{(i)}(x) d x \\
y_{20}(1)\left(1-c_{1}(1)\right) & y_{30}(1)-\int_{0}^{1} \sum_{i=0}^{1} c_{i}(x) y_{30}^{(i)}(x) d x
\end{array}\right|, \\
& M_{22}=\left|\begin{array}{cc}
1+a_{1}(0) & 1-\int_{0}^{1} \sum_{i=0}^{1} a_{i}(x) y_{30}^{(i)}(x) d x \\
c_{1}(0) & y_{30}(1)-\int_{0}^{1} \sum_{i=0}^{1} c_{i}(x) y_{30}^{(i)}(x) d x
\end{array}\right|, \\
& M_{23}=\left|\begin{array}{cc}
1+a_{1}(0) & -a_{1}(1) y_{20}(1) \\
c_{1}(0) & y_{20}(1)\left(1-c_{1}(1)\right)
\end{array}\right|, \\
& M_{31}=\left|\begin{array}{cc}
-a_{1}(1) y_{20}(1) & 1-\int_{0}^{1} \sum_{i=0}^{1} a_{i}(x) y_{30}^{(i)}(x) d x \\
-b_{1}(1) y_{20}(1) & y_{30}^{\prime}(0)-\int_{0}^{1} \sum_{i=0}^{1} b_{i}(x) y_{30}^{(i)}(x) d x
\end{array}\right| .
\end{aligned}
$$


From (11) we obtain the following asymptotic estimations

$$
\begin{gathered}
\left|\Phi_{1}^{(j)}(t, \varepsilon)\right| \leq C+\frac{C}{\varepsilon^{j-1}} e^{-\gamma_{1} \frac{t}{\varepsilon}}+\frac{C}{\varepsilon^{j}} e^{-\gamma_{2} \frac{1-t}{\varepsilon}}, j=\overline{0,2}, \\
\left|\Phi_{2}^{(j)}(t, \varepsilon)\right| \leq C \varepsilon+\frac{C}{\varepsilon^{j-1}} e^{-\gamma_{1} \frac{t}{\varepsilon}}+\frac{C}{\varepsilon^{j-1}} e^{-\gamma_{2} \frac{1-t}{\varepsilon}}, j=\overline{0,2} \\
\left|\Phi_{3}^{(j)}(t, \varepsilon)\right| \leq C+\frac{C}{\varepsilon^{j-1}} e^{-\gamma_{1} \frac{t}{\varepsilon}}+\frac{C}{\varepsilon^{j}} e^{-\gamma_{2} \frac{1-t}{\varepsilon}}, j=\overline{0,2} .
\end{gathered}
$$

Main result. We seek the solution of the problem (1), (2) in the form

$$
y(t, \varepsilon)=\sum_{i=1}^{3} C_{i} \Phi_{i}(t, \varepsilon)+\frac{1}{\varepsilon^{2}} \int_{0}^{t} K_{0}(t, s, \varepsilon) F(s) d s-\frac{1}{\varepsilon^{2}} \int_{t}^{1} K_{1}(t, s, \varepsilon) F(s) d s,
$$

where $\Phi_{i}(t, \varepsilon), i=\overline{1,3}$ are boundary functions, $K_{0}(t, s, \varepsilon), K_{1}(t, s, \varepsilon)$ are auxiliary functions expressed by formula (6), $C_{i}, i=\overline{1,3}$ are unknown constants.

Now, we determine the unknown constants $C_{i}, i=\overline{1,3}$ in (13). For determining these constants we substitute (13) into (2). Then, taking into account (8), we find that

$$
C_{1}=\alpha_{0}-h_{1} P(t, \varepsilon), \quad C_{2}=\alpha_{1}-h_{2} P(t, \varepsilon), \quad C_{3}=\beta-h_{3} P(t, \varepsilon)
$$

where

$$
P(t, \varepsilon)=\frac{1}{\varepsilon^{2}} \int_{0}^{t} K_{0}(t, s, \varepsilon) F(s) d s-\frac{1}{\varepsilon^{2}} \int_{t}^{1} K_{1}(t, s, \varepsilon) F(s) d s .
$$

The effect on the operator $h_{1}$ to function $P(t, \varepsilon)$ is characterized by the following expression

$$
\begin{aligned}
& h_{1} P(t, \varepsilon) \equiv P(0, \varepsilon)-\int_{0}^{1} \sum_{i=0}^{1} a_{i}(x) P^{(i)}(x, \varepsilon) d x=-\frac{1}{\varepsilon^{2}} \int_{0}^{1} K_{1}(0, s, \varepsilon) F(s) d s- \\
& -\int_{0}^{1} a_{0}(x)\left(\frac{1}{\varepsilon^{2}} \int_{0}^{x} K_{0}(x, s, \varepsilon) F(s) d s-\frac{1}{\varepsilon^{2}} \int_{x}^{1} K_{1}(x, s, \varepsilon) F(s) d s\right) d x- \\
& -\int_{0}^{1} a_{1}(x)\left(\frac{1}{\varepsilon^{2}} \int_{0}^{x} K_{0}^{\prime}(x, s, \varepsilon) F(s) d s-\frac{1}{\varepsilon^{2}} \int_{x}^{1} K_{1}^{\prime}(x, s, \varepsilon) F(s) d s\right) d x= \\
& =-\frac{1}{\varepsilon^{2}} \int_{0}^{1} K_{1}(0, s, \varepsilon) F(s) d s-\int_{0}^{1} F(s)\left(\int_{s}^{1} \frac{1}{\varepsilon^{2}} \sum_{i=0}^{1} a_{i}(x) K_{0}^{(i)}(x, s, \varepsilon) d x-\right. \\
& \left.\quad-\int_{0}^{s} \frac{1}{\varepsilon^{2}} \sum_{i=0}^{1} a_{i}(x) K_{1}^{(i)}(x, s, \varepsilon) d x\right) d s=-\frac{1}{\varepsilon^{2}} \int_{0}^{1}\left(K_{1}(0, s, \varepsilon)+\right. \\
& \left.+\int_{s}^{1} \sum_{i=0}^{1} a_{i}(x) K_{0}^{(i)}(x, s, \varepsilon) d x-\int_{0}^{s} \sum_{i=0}^{1} a_{i}(x) K_{1}^{(i)}(x, s, \varepsilon) d x\right) F(s) d s .
\end{aligned}
$$


Then from (14) the constant $C_{1}$ defined by the formula

$$
C_{1}=\alpha_{0}+\frac{1}{\varepsilon^{2}} \int_{0}^{1}\left(K_{1}(0, s, \varepsilon)+\int_{s}^{1} \sum_{i=0}^{1} a_{i}(x) K_{0}^{(i)}(x, s, \varepsilon) d x-\int_{0}^{s} \sum_{i=0}^{1} a_{i}(x) K_{1}^{(i)}(x, s, \varepsilon) d x\right) F(s) d s .
$$

Using formula (7) to (16), we get for the constant $C_{1}$ the following asymptotic estimation as $\varepsilon \rightarrow 0$ :

$$
C_{1}=\alpha_{0}+\int_{0}^{1}\left(a_{1}(s)+\int_{s}^{1} \sum_{i=0}^{1} a_{i}(x) \frac{y_{30}^{(i)}(x) d x}{y_{30}(s)}\right) \frac{F(s)}{A_{1}(s)} d s+O(\varepsilon) .
$$

In this way, the effect on the operators $h_{2}, h_{3}$ to the function $P(t, \varepsilon)$, we define the constants $C_{2}, C_{3}$ :

$$
\begin{gathered}
C_{2}=\alpha_{1}+\frac{F(0)}{\mu_{2}^{2}(0)\left(\mu_{2}(0)-\mu_{1}(0)\right)}+\int_{0}^{1}\left(b_{1}(s)+\int_{s}^{1} \sum_{i=0}^{1} b_{i}(x) \frac{y_{30}^{(i)}(x)}{y_{30}(s)} d x\right) \frac{F(s)}{A_{1}(s)} d s+O(\varepsilon), \\
C_{3}=\beta-\int_{0}^{1}\left(\frac{y_{30}(1)}{y_{30}(s)}+c_{1}(s)-\int_{s}^{1} \sum_{i=0}^{1} c_{i}(x) \frac{y_{30}^{(i)}(x)}{y_{30}(s)} d x\right) \frac{F(s)}{A_{1}(s)} d s+O(\varepsilon) .
\end{gathered}
$$

Substituting (7) into (15), we have the asymptotic representation of the function $P^{(j)}(t, \varepsilon), j=\overline{0,2}$ as $\varepsilon \rightarrow 0$ :

$$
\begin{gathered}
P^{(j)}(t, \varepsilon)=\frac{\mu_{1}^{j-2}(t)-\mu_{2}^{j-2}(t)}{\varepsilon^{j-1}\left(\mu_{2}(t)-\mu_{1}(t)\right)} F(t)+\int_{0}^{t} \frac{y_{30}^{(j)}(t) F(s)}{A_{1}(s) y_{30}(s)} d s- \\
-\frac{\mu_{1}^{j}(t) y_{10}(t) F(0)}{\varepsilon^{j-1} \mu_{1}^{2}(0)\left(\mu_{2}(0)-\mu_{1}(0)\right)} e^{\frac{1}{\varepsilon} \int_{0}^{t} \mu_{1}(x) d x}+\frac{\mu_{2}^{j}(t) y_{20}(t) F(1)}{\varepsilon^{j-1} \mu_{2}^{2}(1) y_{20}(1)\left(\mu_{2}(1)-\mu_{1}(1)\right)} e^{-\frac{1}{\varepsilon} \int_{t}^{1} \mu_{2}(x) d x}+O(\varepsilon), \quad j=\overline{0,2} .
\end{gathered}
$$

Thus, the following theorem holds.

Theorem 1. Let the conditions $(\mathrm{C} 1)-(\mathrm{C} 3)$ are valid. Then integral boundary value problem (1), (2) on the interval $[0,1]$ has an unique solution and expressed by the formula

$$
y(t, \varepsilon)=\sum_{i=1}^{3} C_{i} \Phi_{i}(t, \varepsilon)+P(t, \varepsilon),
$$

where $\Phi_{i}(t, \varepsilon), i=\overline{1,3}$ are boundary functions, $P(t, \varepsilon)$ is defined by the formula (15), $C_{i}, i=\overline{1,3}$ have the form (14) and are expressed by the asymptotic formulas (17), (18), (19).

Theorem 2. If conditions (C1)-(C3) are valid, then solution for integral boundary value problem (1), (2) hold the following asymptotic estimates as $\varepsilon \rightarrow 0$ :

$$
\begin{gathered}
\left|y^{(j)}(t, \varepsilon)\right| \leq C\left(\left|\alpha_{0}\right|+\varepsilon\left|\alpha_{1}\right|+|\beta|+\max _{0 \leq t \leq 1}|F(t)|\right)+\frac{C}{\varepsilon^{j-1}}\left|\mu_{1}^{j-2}(t)-\mu_{2}^{j-2}(t)\right| \max _{0 \leq t \leq 1}|F(t)|+ \\
+\frac{C}{\varepsilon^{j-1}}\left(\left|\alpha_{0}\right|+\left|\alpha_{1}\right|+|\beta|+\max _{0 \leq t \leq 1}|F(t)|\right) e^{-\gamma_{1} \frac{t}{\varepsilon}}+ \\
+\frac{C}{\varepsilon^{j}}\left(\left|\alpha_{0}\right|+\varepsilon\left|\alpha_{1}\right|+|\beta|+\max _{0 \leq t \leq 1}|F(t)|\right) e^{-\gamma_{2} \frac{1-t}{\varepsilon}}, j=\overline{0,2},
\end{gathered}
$$

where $C>0$ is a constant independent of $\varepsilon$. 
Proof. By applying formulas (17)-(19), (12), (20) in (21), we get asymptotic representations of the solution of the problem (1), (2) as $\varepsilon \rightarrow 0$ :

$$
\begin{aligned}
& y^{(j)}(t, \varepsilon)=\left(\alpha_{0}+\int_{0}^{1}\left(a_{1}(s)+\int_{s}^{1} \sum_{i=0}^{1} a_{i}(x) \frac{y_{30}^{(i)}(x)}{y_{30}(s)} d x\right) \frac{F(s)}{A_{1}(s)} d s+O(\varepsilon)\right) . \\
& \cdot\left(\frac{\mu_{1}^{j}(t) y_{10}(t) M_{11}}{\varepsilon^{j-1} \cdot \mu_{1}(0) \bar{\Delta}} e^{\frac{1}{\varepsilon} \int_{0}^{t} \mu_{1}(x) d x}+\frac{\mu_{2}^{j}(t) y_{20}(t)\left(y_{30}(1)+\int_{0}^{1} \sum_{i=0}^{1} c_{i}(x) y_{30}^{(i)}(x) d x\right)}{\varepsilon^{j} \cdot \bar{\Delta}} e^{-\frac{1}{\varepsilon} \int_{t}^{1} \mu_{2}(x) d x}+\right. \\
& \left.+\frac{y_{20}(1) y_{30}^{(j)}(t)\left(1-c_{1}(1)\right)}{\bar{\Delta}}+O\left(\varepsilon+\frac{1}{\varepsilon^{j-2}} e^{\frac{1}{\varepsilon} \int_{0}^{t} \mu_{1}(x) d x}+\frac{1}{\varepsilon^{j-1}} e^{-\frac{1}{\varepsilon} \int_{t}^{1} \mu_{2}(x) d x}\right)\right)+ \\
& +\left(\alpha_{1}+\frac{F(0)}{\mu_{2}^{2}(0)\left(\mu_{2}(0)-\mu_{1}(0)\right)}+\int_{0}^{1}\left(b_{1}(s)+\int_{s}^{1} \sum_{i=0}^{1} b_{i}(x) \frac{y_{30}^{(i)}(x)}{y_{30}(s)} d x\right) \frac{F(s)}{A_{1}(s)} d s+O(\varepsilon)\right) . \\
& \cdot\left(-\frac{\mu_{1}^{j}(t) y_{10}(t) M_{21}}{\varepsilon^{j-1} \cdot \mu_{1}(0) \bar{\Delta}} e^{\frac{1}{\varepsilon} \int_{0}^{t} \mu_{1}(x) d x}+\frac{\mu_{2}^{j}(t) y_{20}(t) M_{22}}{\varepsilon^{j-1} \cdot \mu_{1}(0) \bar{\Delta}} e^{-\frac{1}{\varepsilon} \int_{t}^{1} \mu_{2}(x) d x}-\varepsilon \frac{M_{23} y_{30}^{(j)}(t)}{\mu_{1}(0) \bar{\Delta}}+\right. \\
& \left.+O\left(\varepsilon^{2}+\frac{1}{\varepsilon^{j-2}} e^{\frac{1}{\varepsilon} \int_{0}^{t} \mu_{1}(x) d x}+\frac{1}{\varepsilon^{j-1}} e^{-\frac{1}{\varepsilon} \int_{t}^{1} \mu_{2}(x) d x}\right)\right)+ \\
& +\left(\beta-\int_{0}^{1}\left(\frac{y_{30}(1)}{y_{30}(s)}-c_{1}(s)-\int_{s}^{1} \sum_{i=0}^{1} c_{i}(x) \frac{y_{30}^{(i)}(x)}{y_{30}(s)} d x\right) \frac{F(s)}{A_{1}(s)} d s+O(\varepsilon)\right) . \\
& \cdot\left(\frac{\mu_{1}^{j}(t) y_{10}(t) M_{31}}{\varepsilon^{j-1} \cdot \mu_{1}(0) \bar{\Delta}} e^{\frac{1}{\varepsilon} \int_{0}^{t} \mu_{1}(x) d x}+\frac{\mu_{2}^{j}(t) y_{20}(t)\left(1-\int_{0}^{1} \sum_{i=0}^{1} a_{i}(x) y_{30}^{(i)}(x) d x\right)}{\varepsilon^{j} \cdot \bar{\Delta}} e^{-\frac{1}{\varepsilon} \int_{t}^{1} \mu_{2}(x) d x}+\right. \\
& \left.+\frac{a_{1}(1) y_{20}(1) y_{30}^{(j)}(t)}{\bar{\Delta}}+O\left(\varepsilon+\frac{1}{\varepsilon^{j-2}} e^{\frac{1}{\varepsilon} \int_{0}^{t} \mu_{1}(x) d x}+\frac{1}{\varepsilon^{j-1}} e^{-\frac{1}{\varepsilon} \int_{t}^{1} \mu_{2}(x) d x}\right)\right)+\int_{0}^{t} \frac{y_{30}^{(j)}(t) F(s)}{A_{1}(s) y_{30}(s)} d s+ \\
& +\frac{\mu_{1}^{j-2}(t)-\mu_{2}^{j-2}(t)}{\varepsilon^{j-1}\left(\mu_{2}(t)-\mu_{1}(t)\right)} F(t)-\frac{\mu_{1}^{j}(t) y_{10}(t) F(0)}{\varepsilon^{j-1} \cdot \mu_{1}^{2}(0)\left(\mu_{2}(0)-\mu_{1}(0)\right)} e^{\frac{1}{\varepsilon} \int_{0}^{t} \mu_{1}(x) d x}+ \\
& \frac{\mu_{2}^{j}(t) y_{20}(t) F(1)}{\varepsilon^{j-1} \cdot \mu_{1}^{2}(1) y_{20}(1)\left(\mu_{2}(1)-\mu_{1}(1)\right)} e^{-\frac{1}{\varepsilon} \int_{t}^{1} \mu_{2}(x) d x}+O(\varepsilon) .
\end{aligned}
$$

From asymptotic representations (23), we obtain asymptotic estimations (22). Theorem 2 is proved.

The theorem 2 implies that the solution of the problem (1), (2) at point $t=0$ has the phenomenon of the first order initial jump and at point $t=1$ has the phenomenon of the zero order initial jump, i.e.

$$
y(0, \varepsilon)=O(1), y^{\prime}(0, \varepsilon)=O(1), y^{\prime \prime}(0, \varepsilon)=O\left(\frac{1}{\varepsilon}\right)
$$


and

$$
y(1, \varepsilon)=O(1), y^{\prime}(1, \varepsilon)=O\left(\frac{1}{\varepsilon}\right), y^{\prime \prime}(1, \varepsilon)=O\left(\frac{1}{\varepsilon^{2}}\right) .
$$

In this case, we say that the solution of the boundary value problem (1), (2) has the phenomenon of the boundary jumps.

\section{Conclusion}

In this paper, we consider a three-point boundary value problem for a third-order linear differential equation with a small parameter at two highest derivatives when the roots of the "additional characteristic equation" have negative signs. Theorem about asymptotic estimates of solution is proved. It is established that the solution of this integral boundary value problem has the phenomenon of boundary jumps. This means that the points of the initial jump are not only the left, but also the right point of the segment. The results allow us to construct uniform asymptotic expansions of solutions of boundary value problems with boundary jumps with any degree of accuracy with respect to a small parameter.

\section{Acknowledgement}

The authors were supported in parts by the MESRK grants AP05132587 «Boundary value problems for singularly perturbed differential equations with a continuous and piecewise constant argument» (2018-2020) and AP05132573 «Cellular neural networks with continuous/discrete time and singular perturbations» (2018-2020) of the Committee of Science, Ministry of Education and Science of the Republic of Kazakhstan.

\section{References}

1 Schlesinger L. Uber asymptotische darstellungen der losungen linearer differential systeme als funktionen eines parameters / L. Schlesinger // Mathematische Annalen. - 1907. - Vol. 63, No. 3. - P. 277-300.

2 Birkhoff G.D. On the asymptotic character of the solutions of certain linear differential equations containing a parameter / G.D. Birkhoff // Transactions of the American Mathematical Society. - 1908. - Vol. 9, No. 2. - P. 219-231.

3 Noaillon Developpements asymptotiques dans les equations differentielles lineaires a parameter variable / Noaillon // Mem. Soc. Sci. Liege. - 1912. - Vol. 3, No. 11. - P. 197.

4 Wasow W. Singular perturbations of boundary value problems for nonlinear differential equations of the second order / W. Wasow // Comm. On Pure and Appl. Math. - 1956. - No. 9. - P. 93113.

5 Тихонов А.Н. О зависимости решений дифференциальных уравнений от малого параметра / А.Н. Тихонов // Матем. сб. - 1948. - Т. 22, Вып. 2. - С. 193-204.

6 Тихонов А.Н. О системах дифференциальных уравнений, содержащих параметры / А.Н. Тихонов // Матем. сб. - 1950. - Т. 27, Вып. 69. - С. 147-156.

7 Vishik M.I. Regular degeneration and boundary layer for linear differential equations with small parameter multiplying the highest derivatives / M.I. Vishik, L.A. Lyusternik // Usp. Mat. Nauk. - 1957. - No. 12. - P. 3-122; Amer. Math. Soc. Transl. - 1962. - Vol. 20, No. 2. - P. 239-364.

8 Vishik M.I. On the initial jump for non-linear differential equations containing a small parameter / M.I. Vishik, L.A. Lyusternik // Doklady Akademii Nauk SSSR. - 1960. - Vol. 132, No. 6. P. 1242-1245.

9 Bogoliubov N.N. The method of integral manifolds in nonlinear mechanics, In Contributions to Differential Equations / N.N. Bogoliubov, Yu.A. Mitropolsky // Interscience-John Wiley. - 1963. - No. 2. - P. 123-196. 
10 Vasil'eva A.B. The boundary function method for singular perturbation problems / A.B. Vasil'eva, A.B. Butuzov, L. Kalachev // SIAM Studiesin Applied Mathematics. - Philadeplhia, 1995.

11 Trenogin V.A. Development and applications of the asymptotic method of Liusternik and Vishik / V.A. Trenogin // Usp. Mat. Nauk. - 1970. - No. 25. - P. 123-156; Transl. in Russ. Math. Surv. - No. 25. - P. 119-156.

12 O'Malley R. Singular perturbations methods for ordinary differential equations / R. O'Malley. - Berlin, Heidelberg: Springer-Verlag, 1991.

13 Eckhaus W. Fundamental concepts of matching / W. Eckhaus // SIAM Rev. - 1994. - No. 36. - P. 431-439.

14 Chang K. Nonlinear Singular Perturbation Phenomena: Theory and Application / K. Chang, K. Howes. - New York: Springer-Verlag, 1984.

15 Kevorkian J. Singular perturbation methods in applied mathematics / J. Kevorkian, J.D. Cole. - Berlin, Heidelberg: Springer-Verlag, 1981.

16 Kokotovic P.V. Applications of singular perturbation techniques to control problems / P.V. Kokotovic // SIAM Rev. - 1984. - No. 26. - P. 501-550.

17 Lomov S.A. Introduction to the general theory of singular perturbations / S.A. Lomov. American Mathematical Society, Providence, 1992.

18 Иманалиев М.И. Асимптотические методы в теории сингулярно возмущенных интегродифференциальных систем / М.И. Иманалиев. - Фрунзе: Илим, 1972.

19 Касымов К.А. Об асимптотике решения задачи Коши с большими начальными условиями для нелинейного обыкновенного дифференциального уравнения, содержащего малый параметр / К.А. Касымов // Успехи математических наук. - 1962. - Т. 17, Вып. 5. - Р. 187-188.

20 Касымов К.А. О задаче с начальным скачком для нелинейных систем дифференциальных уравнений, содержащих малый параметр / К.А. Касымов // Докл. АН СССР. - 1968. Т. 179, Вып. 2. - Р. 275-278.

21 Касымов К.А. Асимптотика решения задачи с начальными скачками для системы дифференциальных уравнений гиперболического типа с малым параметром при производной / К.А. Касымов // Докл. АН СССР. - 1971. - Т. 196, Вып. 2. - С. 274-277.

22 Абильдаев Е.А. Асимптотические оценки решений сингулярно возмущенных краевых задач с начальными скачками для линейных дифференциальных уравнений / Е.А. Абильдаев, К.А. Касымов // Дифференциальные уравнения. - 1992. - Т. 28, Вып. 10. - Р. 1659-1668.

23 Kassymov K.A. Estimates of solutions of the Cauchy problem with an arbitrary-order initial jump for linear singularly perturbed integro-differential equations / K.A. Kassymov, M.K. Dauylbaev // Differential Equations. - 1999. - Vol. 35, No. 6. - P. 822-830.

24 Dauylbaev M.K. The asymptotic behavior of solutions to singularly perturbed nonlinear integrodifferential equations / M.K. Dauylbaev // Siberian Mathematical Journal. - 2000. - Vol. 41, No. 1. - P. 49-60.

25 Kassymov K.A. Asymptotic behavior of solutions of linear singularly perturbed general separated boundary-value problems with initial jump / K.A.Kassymov, D.N. Nurgabyl // Ukrainian Mathematical Journal. - 2003. - Vol. 55, No. 11. - P. 1777-1792.

26 Kassymov K.A. Asymptotic estimates of solution of a singularly perturbed boundary value problem with an initial jump for linear differential equations / K.A.Kassymov, D.N. Nurgabyl // Differential Equations. - 2004. - Vol. 40, No. 5. - P. 641-651.

27 Kassymov K.A. Asymptotic estimates for the solutions of boundary-value problems with initial jump for linear differential equations with small parameter in the coefficients of derivatives / K.A.Kassymov, D.N. Nurgabyl, A.B. Uaissov // Ukrainian Mathematical Journal. - 2013. Vol. 65, No. 5. - P. 694-708. 
28 Nurgabul D. Asymptotic estimates for the Solution of a Restoration Problem with Initial Jump / D. Nurgabul // Journal of Applied Mathematics.- Vol.2014. - Article ID 956402.

29 Dauylbayev M.K. The initial jumps of solutions and integral terms in singular BVP of linear higher order integro-differential equations / M.K. Dauylbayev, N. Atakhan // Miskolc Math. Notes. - 2015. - Vol. 16, No. 2. - P. 747-761.

30 Akhmet M. A singularly perturbed differential equation with piecewise constant argument of generalized type / M. Akhmet, M. Dauylbayev, A. Mirzakulova // Turkish Journal of Mathematics. - 2018. - Vol. 42, No. 4. - P. 1680 - 1685.

31 Касымов К.А. Асимптотические оценки решения краевой задачи для сингулярно возмущенных линейных дифференциальных уравнений третьего порядка / К.А. Касымов, Ж.У. Шарипова // Вестн. КазГУ им. С.М. Кирова. Сер. мат. - 1993. - Вып. 1. - Р. 146-150.

32 Касымов К.А. Представление решения краевой задачи для линейного дифференциального уравнения с малым параметром при старших производных / К.А. Касымов, Д.А. Жакыпбекова, Д.Н. Нургабыл // Вестн. КазНУ им. аль-Фараби. Сер. мат., мех., инф. - 2001. Вып. 3. - Р. 73-78.

33 Dauylbaev M.K. Boundary-value problems with initial jumps for singularly perturbed integrodifferential equations / M.K. Dauylbaev, A.E. Mirzakulova // Journal of Mathematical Sciences. - 2017. - Vol. 222, No. 3. - P. 214-225.

Н.Ұ. Буканай, А.Е. Мирзакулова, М.Қ. Дауылбаев, К.Т. Қонысбаева

\title{
Сингулярлы ауытқыған дифференциалдық теңдеулерге арналған интегралдық шеттік есептегі шекаралық секірістер құбылысы
}

\begin{abstract}
Мақала қосымша сипаттаушы теңдеудің түбірлері қарама-қарсы болған жағдайдағы екі жоғарғы туындыларының алдында кіші параметрі бар үшінші ретті сызықты дифференциалдық теңдеу үшін шекаралы секірісті жалпы интегралды шеттік есебін зерттеуге арналған. Жұмыста қосымша сипаттаушы теңдеудің түбірлері қарама-қарсы болған жағдайдағы сингулярлы ауытқыған біртекті дифференциалдық теңдеудің іргелі шешімдер жүйесі құрылған. Іргелі шешімдер жүйесі арқылы сингулярлы ауытқыған біртекті дифференциалдық теңдеудің $K_{i}(t, s, \varepsilon), i=0,1$ көмекші функциялары және шекаралық функциялары берілген. Және олардың асимптотикалық сипаттары мен бағалаулары келтірілген. Берілген сингулярлы ауытқыған жалпы интегралды шеттік есеп шешімінің аналитикалық формуласы алынған. Шешімнің асимптотикалық бағалауы туралы теорема дәлелденген. Сингулярлы ауытқыған жалпы интегралды шеттік есеп шешімі кесіндінің екі жақ шетінде де бастапқы секіріске ие болатыны анықталған. Зерттеу нәтижесінде есеп шешімінің сол жақ және оң жақ нүктелерінде әртүрлі ретті бастапқы секіріс құбылыстарын және алынған нәтижелердің қорытындысында берілген шеттік есептің шешімінің $t=0$ нүктесінде бірінші ретті, ал $t=1$ нүктесінде нөлінші ретті бастапқы секірістері бар екендігі анықталды. Алынған нәтижелер сызықты емес сингулярлы ауытқыған интегралды шеттік есептер шешімдерінің біркелкі асимптотикалық жіктелуін құруға мүмкіндік береді.

Kiлm сөздер: сингулярлы ауытқыған дифференциалдық теңдеу, асимптотикалық бағалау, шекаралық функциялар, кіші параметр.
\end{abstract}


Н.У. Буканай, А.Е. Мирзакулова, М.К. Дауылбаев, К.Т. Конысбаева

\title{
Явление граничных скачков в интегральной краевой задаче для сингулярно возмущенных дифференциальных уравнений
}

\begin{abstract}
Статья посвящена исследованию асимптотического поведения решения интегральной краевой задачи для линейного дифференциального уравнения третьего порядка с малым параметром при двух старших производных при условии, когда корни «дополнительного характеристического уравнения» имеют противоположные знаки. В работе построена фундаментальная система решений сингулярно возмущенного однородного дифференциального уравнения с учетом противоположности знаков корней «дополнительного характеристического уравнения». Затем с помощью фундаментальной системы решений строятся вспомогательные функции и граничные функции сингулярно возмущенного однородного дифференциального уравнения. Получены асимптотические представления и оценки вспомогательных и граничных функций. Получена аналитическая формула решения рассматриваемой сингулярно возмущенной интегральной краевой задачи. Доказана теорема об асимптотических оценках решения. Для сингулярно возмущенной интегральной краевой задачи получен рост решения и его производных в граничных точках данного отрезка при стремлении малого параметра к нулю. Установлено, что решение сингулярно возмущенной интегральной краевой задачи имеет начальные скачки на обоих концах данного отрезка. В этом случае мы говорим, что имеет место явление граничных скачков, что является особенностью рассматриваемой сингулярно возмущенной интегральной краевой задачи. Причем порядки начальных скачков оказались разными. А именно: в точке $t=0$ имеет место явление начального скачка первого порядка, а в точке $t=1$ порядок начального скачка оказался равным нулю. Полученные результаты позволяют построить равномерные асимптотические разложения решений нелинейных сингулярно возмущенных интегральных краевых задач.
\end{abstract}

Ключевые слова: сингулярно возмущенное дифференциальное уравнение, асимптотические оценки, граничные функции, малый параметр.

\section{References}

1 Schlesinger, L. (1907). Uber asymptotische darstellungen der losungen linearer differential systeme als funktionen eines parameters. Mathematische Annalen, 63(3), 277-300.

2 Birkhof, G.D. (1908). On the asymptotic character of the solutions of certain linear differential equations containing a parameter. Transactions of the American Mathematical Society, 9(2), 219-231.

3 Noaillon. (1912). Developpements asymptotiques dans les equations differentielles lineaires a parameter variable. Mem. Soc. Sci. Liege., 3(11), 197.

4 Wasow, W. (1956). Singular perturbations of boundary value problems for nonlinear differential equations of the second order. Comm. On Pure and Appl. Math., 9, 93-113.

5 Tikhonov, A.N. (1948). O zavisimosti reshenii differentsialnykh uravnenii ot maloho parametra. Matematicheskii sbornik, 22(2), 193-204 [in Russian].

6 Tikhonov, A.N. (1950). O sistemakh differentsialnykh uravnenii, soderzhashchikh parametry. Matematicheskii sbornik, 27(69), 147-156 [in Russian].

7 Vishik, M.I., \& Liusternik, L.A. (1957, 1962). Regular degeneration and boundary layer for linear differential equations with small parameter multiplying the highest derivatives. Usp. Mat. Nauk, 12, 3-122 [in Russian]; Amer. Math. Soc. Transl., 20, 2, 239-364.

8 Vishik, M. I., \& Liusternik, L.A. On the initial jump for non-linear differential equations containing a small parameter. Doklady Akademii Nauk SSSR, 132(6), 1242-1245.

9 Bogoliubov, N.N., \& Mitropolsky, Yu.A. (1963). The method of integral manifolds in nonlinear mechanics, In Contributions to Differential Equations. Interscience-John Wiley, 2, 123-196.

10 Vasil'eva, A. B., Butuzov, A. B., \& Kalachev, L. (1995). The boundary function method for singular perturbation problems. SIAM Studiesin Applied Mathematics, Philadeplhia. 
11 Trenogin, V.A. (1970). Development and applications of the asymptotic method of Liusternik and Vishik. Usp. Mat. Nauk, 25, 123-156; Transl. in Russ. Math. Surv. 25, 119-156.

12 O'Malley, R. (1991). Singular perturbations methods for ordinary differential equations. Berlin, Heidelberg: Springer-Verlag.

13 Eckhaus, W. (1994). Fundamental concepts of matching. SIAM Rev., 36, 431-439.

14 Chang, K., \& Howes, K. (1984). Nonlinear Singular Perturbation Phenomena: Theory and Application. New York: Springer Verlag.

15 Kevorkian, J., \& Cole, J.D. (1981). Singular perturbation methods in applied mathematics. Berlin, Heidelberg: Springer-Verlag.

16 Kokotovic P.V. (1984). Applications of singular perturbation techniques to control problems. SIAM Rev, 26, 501-550.

17 Lomov, S. A. (1992). Introduction to the general theory of singular perturbations. American Mathematical Society, Providence.

18 Imanaliev, M.I. (1972). Asimptoticheskie metody v teorii sinhuliarno vozmushchennykh intehrodifferentsialnykh sistem. Frunze: Ilim [in Russian].

19 Kassymov, K.A. (1962). Ob asimptotike resheniia zadachi Koshi s bolshimi nachalnymi usloviiami dlia nelineinoho obyknovennoho differentsialnoho uravneniia, soderzhashcheho malyi parametr. Uspekhi matematicheskikh nauk, 17(5), 187, 188 [in Russian].

20 Kassymov, K.A. (1968). O zadache s nachalnym skachkom dlia nelineinykh sistem differentsialnykh uravnenii, soderzhashchikh malyi parametr. DAN SSSR, 179(2), 275-278 [in Russian].

21 Kassymov, K.A. (1971). Asimptotika resheniia zadachi s nachalnymi skachkami dlia sistemy differentsialnykh uravnenii hiperbolicheskoho tipa s malym parametrom pri proizvodnoi. DAN SSSR, 196(2), 274-277 [in Russian].

22 Abildaev, E.A., \& Kassymov, K.A. (1992). Asimptoticheskie otsenki reshenii sinhuliarno vozmuschennykh kraevikh zadach s nachalnimi skachkami dlia lineinikh differentsialnikh uravnenii. Differentsialnie uravneniia, 28(10), 1659-1668 [in Russian].

23 Kassymov, K.A., \& Dauylbaev, M.K. (1999). Estimates of solutions of the Cauchy problem with an arbitrary-order initial jump for linear singularly perturbed integro-differential equations. Differential Equations, 35(6), 822-830.

24 Dauylbaev, M.K. (2000). The asymptotic behavior of solutions to singularly perturbed nonlinear integro-differential equations. Siberian Mathematical Journal, 41(1), 49-60.

25 Kassymov, K.A., \& Nurgabyl, D.N. (2003). Asymptotic behavior of solutions of linear singularly perturbed general separated boundary-value problems with initial jump. Ukrainian Mathematical Journal, 55(11), 1777-1792.

26 Kassymov, K.A., \& Nurgabyl, D.N. (2004). Asymptotic estimates of solution of a singularly perturbed boundary value problem with an initial jump for linear differential equations. Differential Equations, 40(5), 641-651.

27 Kassymov, K.A., Nurgabyl D.N., \& Uaissov A.B. (2013). Asymptotic estimates for the solutions of boundary-value problems with initial jump for linear differential equations with small parameter in the coefficients of derivatives. Ukrainian Mathematical Journal, 65(5), 694-708.

28 Nurgabul D. (2014). Asymptotic estimates for the Solution of a Restoration Problem with Initial Jump Journal of Applied Mathematics, Vol.2014, Article ID 956402.

29 Dauylbayev, M.K., \& Atakhan, N. (2015). The initial jumps of solutions and integral terms in singular BVP of linear higher order integro-differential equations. Miskolc Math. Notes, 16( 2), 747-761. 
30 Akhmet, M., Dauylbayev, M., \& Mirzakulova, A. (2018). A singularly perturbed differential equation with piecewise constant argument of generalized type. Turkish Journal of Mathematics, 42(4), 1680-1685.

31 Kassymov, K.A., \& Sharipova, Zh.U. (1993). Asimptoticheskie otsenki resheniia kraevoi zadachi dlia sinhuliarno vozmushchennykh lineinykh differentsialnykh uravnenii treteho poriadka. Vestnik KazHU imeni S.M. Kirova. Seriia matematicheskaia, 1, 146-150 [in Russian].

32 Kassymov, K.A., Zhakipbekova, D.A., \& Nurgabyl, D.N. (2001). Predstavlenie resheniia kraevoi zadachi dlia lineinoho differentsialnoho uravneniha s malym parametrom pri starshikh proizvodnykh. Vestnik KazNU imeni al'-Farabi. Seriia matematika, mekhanika, informatika, 3, 73-78 [in Russian].

33 Dauylbaev, M.K., \& Mirzakulova, A.E. (2017). Boundary-value problems with initial jumps for singularly perturbed integro-differential equations. Journal of Mathematical Sciences, 222( 3), 214-225. 